\title{
OPEN Soluble PD-L1 is a predictive and prognostic biomarker in advanced cancer patients who receive immune checkpoint blockade treatment
}

\author{
So Yeon $\mathrm{Oh}^{1}$, Soyeon $\mathrm{Kim}^{2}$, Bhumsuk Keam ${ }^{3,4}$, Tae Min Kim ${ }^{3,4}$, Dong-Wan Kim ${ }^{2,4}$ \& \\ Dae Seog $\mathrm{HeO}^{3,4 \bowtie}$
}

Circulating soluble programmed death-1 ligand (SPD-L1) is measurable in the serum of cancer patients. This study aimed to investigate the significance of SPD-L1 in cancer patients receiving immune checkpoint inhibitor therapy. Blood samples were obtained before and after immune checkpoint inhibitor therapy (January 2015 to January 2019). The study cohort consisted of 128 patients who were diagnosed with non-small cell lung cancer $(n=50)$, melanoma $(n=31)$, small cell lung cancer $(n=14)$, urothelial carcinoma $(n=13)$, and other cancers $(n=20)$. Patients with a high level $(>11.0 \mathrm{pg} / \mu \mathrm{L}$ ) of sPD-L1 were more likely to exhibit progressive disease compared with those with a low level ( $41.8 \%$ versus $20.7 \%, p=0.013)$. High SPD-L1 was also associated with worse prognosis; the median PFS was 2.9 (95\% confidence interval $[\mathrm{Cl}] 2.1-3.7)$ months versus $6.3(95 \% \mathrm{Cl} 3.0-9.6)$ months $(p=0.023)$, and the median OS was $7.4(95 \% \mathrm{Cl} 6.3-8.5)$ months versus $13.3(95 \% \mathrm{Cl} 9.2-17.4)$ months $(p=0.005)$. In the multivariate analyses, high sPD-L1 was an independent prognostic factor for both decreased PFS (HR 1.928, $p=0.038$ ) and OS (HR 1.788, $p=0.004)$. sPD-L1 levels did not correlate with tissue PD-L1 expression. However, SPD-L1 levels were positively correlated with neutrophil to lymphocyte ratios and negatively correlated with both the proportion and the total number of lymphocytes. We found that high pretreatment SPD-L1 levels were associated with progressive disease and were an independent prognostic factor predicting lower PFS and OS in these patients.

Since the first immune checkpoint inhibitor (ICI), ipilimumab, was approved in 2011, the treatment paradigm for solid tumors has changed greatly. ICIs play important roles in the treatment of various types of solid tumors. However, in clinical practice, $16-55 \%$ of patients receiving ICI treatment suffer from severe toxicities ${ }^{1}$. Therefore, it is important to identify predictors of this negative response. Some biomarkers that predict treatment response have already been identified. For example, programmed death ligand 1 (PD-L1) ${ }^{2}$ expression in tumor tissue is predictive of a higher response rate to programmed death 1 (PD-1) or PD-L1 inhibitor therapy in patients with non-small cell lung cancer (NSCLC) $)^{3-5}$. It is also a marker of poor prognosis in some solid tumors ${ }^{6-8}$. However, inter-assay discordance and tumor heterogeneity hinder the standardization of PD-L1 testing and interpretation ${ }^{9,10}$. Researchers have tried to standardize the methods used to measure PD-L1 expression, but no clinically validated assays are currently available ${ }^{11}$. Microsatellite instability or deficient mismatch repair (dMMR) is one of the biomarkers studied as a predictor of ICI response ${ }^{2}$. dMMR is associated with favorable clinical outcomes in patients with colorectal cancer ${ }^{12,13}$. PD-1 blockade results in a robust response in some subjects with dMMR-positive solid tumors ${ }^{14}$. Tumor mutational burden, tumor infiltrating lymphocytes, and genetic signatures are also predictive factors. However, these markers lack standardization and exhibit high variability across tumor types and study settings ${ }^{2}$.

\footnotetext{
${ }^{1}$ Medical Oncology, Department of Internal Medicine, Pusan National University Yangsan Hospital, Yangsan, Republic of Korea. ${ }^{2}$ Cancer Research Institute, Seoul National University and Integrated Major in Innovative Medical Science, Seoul National University College of Medicine, Seoul, Republic of Korea. ${ }^{3}$ Biomedical Research Institute, Seoul National University, Seoul, Republic of Korea. ${ }^{4}$ Department of Internal Medicine, Seoul National University Hospital, 101, Daehak-ro, Jongno-gu, Seoul 03080, Republic of Korea. ${ }^{\circledR}$ email: heo1013@snu.ac.kr
} 
Lack of sufficient tissue for examination is another common and important factor limiting biomarker utility. A shortage of tumor tissue is especially problematic in patients with NSCLC because diagnostic biopsies often yield only tiny pieces of tissue. However, approximately five molecular genetic tests are required to select the appropriate therapeutic agents for an individual NSCLC patient ${ }^{15,16}$. The use of small biopsies can also result in misclassification of up to $35 \%$ of PD-L1 assessments in patients with advanced NSCLCs ${ }^{17}$. Therefore, circulating blood biomarkers are being investigated to predict the response to PD-1/PD-L1 blockade. These potential markers include, among others, circulating immune cells and circulating PD-L1 ${ }^{18-23}$. In previous studies, CD8 ${ }^{+}$ $\mathrm{T}$ cells showed a proliferative burst or functional reinvigoration after PD-1 blockade ${ }^{24,25}$. Another study showed that functionally active $\mathrm{CD} 8^{+} \mathrm{T}$ cells or NK cells are associated with good prognosis after PD-1 blockade ${ }^{26}$.

PD-L1 is present in a membrane-bound form in tumor cells and immune cells. However, it may also be secreted as a truncated form called soluble PD-L1 (sPD-L1) which may mediate immunosuppression or resistance to PD-L1 blockade therapy ${ }^{27-29}$. Compared with healthy subjects, circulating sPD-L1 concentrations are elevated in the plasma of patients with cancer. In patients with lymphoma, these concentrations return to normal levels after a complete response ${ }^{30}$. High concentrations of sPD-L1 are associated with poor prognosis in patients with hepatocellular carcinoma, gastric cancer, and NSCLC ${ }^{31-34}$. Here, we examined circulating SPD-L1 and its role as a prognostic and predictive marker in patients with cancer who received ICI treatment. We analyzed pretreatment and posttreatment levels of circulating SPD-L1 and investigated the relationship between these levels and clinical outcomes in patients with advanced solid tumors.

\section{Results}

Patient and sample characteristics. A total of 128 patients with stage IV solid tumors were included in this study. Samples were obtained between January 2015 and January 2019. The characteristics of the study population are presented in Table 1. The sample interval range was 14-49 days in 66 of the 67 patients with available pre- and posttreatment samples; in the remaining patient, the sample interval was 576 days (median, 21 days; range, 14-576 days; Supplementary Table 1). The objective response rate (ORR) was 18.8\% (among 112 evaluable patients). The median progression-free survival (PFS) and overall survival (OS) were 4.2 months (95\% CI 2.3-6.1 months) and 10.8 months (95\% CI 7.9-13.8 months), respectively.

Pretreatment sPD-L1 level and response. The mean pretreatment sPD-L1 level was $13.5 \pm 12.1 \mathrm{pg} /$ $\mu \mathrm{L}$ and the median level was $11.0 \mathrm{pg} / \mu \mathrm{L}$ (range, 3.2-122.1 pg/ $\mu \mathrm{L}$ ). The mean sPD-L1 value in the patients with cancer was not significantly different from the mean level in healthy volunteers $(13.5 \mathrm{pg} / \mu \mathrm{L}$ versus $10.6 \mathrm{pg} /$ $\mu \mathrm{L}$, respectively; $\mathrm{p}=0.312, t$ test). However, the mean $\mathrm{sPD}-\mathrm{L} 1$ concentration was numerically higher in cancer patients and we have to consider the possibility that there was no statistical significance because of the small number of healthy volunteers $(n=20)$. We used a receiver operating characteristic (ROC) curve to determine the optimal sPD-L1 cutoff level for the prediction of progressive disease after ICI treatment (Supplementary Fig. 2). We found that a cutoff value of $11.0 \mathrm{pg} / \mu \mathrm{L}$ distinguished best between patients who showed progressive disease after ICI treatment versus those who did not have progressive disease (sensitivity, 65.7\%; specificity, 60.3\%). The area under the curve value was 0.668 (95\% confidence interval $(\mathrm{CI}) 0.568-0.769 ; \mathrm{p}=0.004)$. This cutoff value was used for subsequent statistical analyses. We used $\chi^{2}$ tests to compare treatment response in patients with varying sPD-L1 levels (low versus high). The ORRs were not significantly different ( $23.4 \%$ versus $22.7 \%$ in the low and high groups, respectively; $\mathrm{p}=0.573$ ). However, the disease control rates were $79 \%$ versus $58 \%$ in patients with low and high sPD-L1 levels, respectively $(\mathrm{p}=0.013)$ (Table 2). The relationship between sPD-L1 levels and treatment response is illustrated with a bar chart in Supplementary Fig. 3.

Pretreatment sPD-L1 levels and prognosis. PFS varied between patients with low versus high levels of sPD-L1. The median PFS was 6.3 months (95\% CI 3.0-9.6 months) versus 2.9 months (95\% CI 2.1-3.7 months); this difference was statistically significant $(\mathrm{p}=0.023$, log-rank test; Fig. 1a). OS was also significantly different between the two groups. The median OS was 13.3 months (95\% CI 9.2-17.4 months) versus 7.4 months (95\% CI 6.3-8.5 months) ( $\mathrm{p}=0.005$, log-rank test; Fig. 1b) in the low and high sPD-L1 groups, respectively. Univariate and multivariate analyses were performed to investigate potential correlations between sPD-L1 levels, clinical factors, and PFS and OS (Table 3). The results of the univariate analysis indicated that performance status, tissue PD-L1 expression, neutrophil to lymphocyte ratio (NLR), serum albumin levels, and sPD-L1 levels were significant predictors of PFS. Performance status, NLR, platelet count, serum albumin levels, total serum protein levels, and sPD-L1 levels were significant predictors of OS. The PFS and OS of patients who received radiation prior to ICI therapy were not significantly different from those who did not. Factors with p-values $<0.05$ were included in the multivariate analysis. The multivariate analysis found that tissue PD-L1 expression and sPD-L1 levels were significant predictors of PFS. NLR, total serum protein levels, and sPD-L1 levels were significant predictors of OS (Table 3).

Changes in SPD-L1 levels after treatment. We analyzed the changes in sPD-L1 concentrations in 67 patients with available pre- and posttreatment samples. sPD-L1 levels generally increased with ICI administration during the 2- to 7-week period following the start of treatment. However, the changes in sPD-L1 concentration $(\Delta \mathrm{sPD}-\mathrm{L} 1)$ varied (Fig. $2 \mathrm{a})$, and the patterns of change were somewhat different for each cancer type (Figs. 2b, 3e). A sharp increase was apparent in some patients with NSCLC or genitourinary cancer (Fig. 2b,e). However, with one exception, the amplitude of change was negligible in SCLC and melanoma patients (Fig. 2c,d). For comparison, we analyzed $\Delta \mathrm{sPD}$-L1 between pre- and posttreatment samples from NSCLC patients treated with tyrosine kinase inhibitors. The results indicated that the pattern of change in NSCLC patients treated with tyrosine kinase inhibitors was very similar to that of the SCLC patients (Fig. 2f). Interestingly, the pattern of 


\begin{tabular}{|c|c|}
\hline & $\mathrm{N}(\%)$ or median(range) \\
\hline Total & $128(100)$ \\
\hline Age & $62(21-82)$ years \\
\hline \multicolumn{2}{|l|}{ Sex } \\
\hline Male & $89(69.5)$ \\
\hline Female & $39(30.5)$ \\
\hline \multicolumn{2}{|l|}{ ECOG PS } \\
\hline 0 & $27(21.1)$ \\
\hline 1 & $98(76.6)$ \\
\hline 2 & $1(0.8)$ \\
\hline Unknown & $2(1.6)$ \\
\hline \multicolumn{2}{|l|}{ Diagnosis } \\
\hline NSCLC & $50(39.1)$ \\
\hline Melanoma & $31(24.2)$ \\
\hline SCLC & $14(10.9)$ \\
\hline UCC & $13(10.2)$ \\
\hline RCC & $6(4.7)$ \\
\hline HNSCC & $5(3.9)$ \\
\hline Salivary gland cancer & $4(5.8)$ \\
\hline Others & $5(3.9)$ \\
\hline \multicolumn{2}{|l|}{ History of radiotherapy* } \\
\hline Never irradiated & $50(39.1)$ \\
\hline Received radiotherapy & $78(60.9)$ \\
\hline Within 4 weeks to ICI treatment & $14(17.9)$ \\
\hline Earlier than 4 weeks & $43(55.1)$ \\
\hline After end of ICI treatment & $21(26.9)$ \\
\hline \multicolumn{2}{|l|}{ Type of ICI treated $^{\dagger}$} \\
\hline \multicolumn{2}{|l|}{ Monotherapy } \\
\hline Nivolumab & $41(32.0)$ \\
\hline Pembrolizumab & $32(25.0)$ \\
\hline Durvalumab & $15(11.7)$ \\
\hline Ipilimumab & $5(3.9)$ \\
\hline Atezolizumab & $4(3.1)$ \\
\hline \multicolumn{2}{|l|}{ Combination therapy } \\
\hline Pembrolizumab/other & $13(10.2)$ \\
\hline Atezolizumab/other & $13(10.2)$ \\
\hline Nivolumab and ipilimumab & $2(1.6)$ \\
\hline Others & $3(2.3)$ \\
\hline
\end{tabular}

Table 1. Patient characteristics. ECOG PS eastern cooperative oncology group performance status, NSCLC non-small cell lung cancer, $S C L C$ small cell lung cancer, $U C C$ urothelial carcinoma, $R C C$ renal cell carcinoma, HNSCC head and neck squamous cell carcinoma, ICI immune checkpoint inhibitor. ${ }^{\star}$ Encompasses all types of radiotherapy, including stereotactic radiosurgery. ${ }^{\dagger}$ Denotes patients who received ICI as part of a clinical trial.

\begin{tabular}{|l|l|l|l|l|}
\hline \multirow{2}{*}{} & \multicolumn{2}{|l|}{ ICI response } & \multirow{2}{*}{ Total } & \multirow{2}{*}{ p value } \\
\cline { 2 - 4 } & CR, PR, SD & PD & $58(100)$ & \multirow{2}{*}{0.013} \\
\hline Low sPD-L1 & $46(79)$ & $12(21)$ & $55(100)$ & \\
\hline High sPD-L1 & $32(58)$ & $23(42)$ & 113 & \\
\hline Total & 78 & 35 & & \\
\hline
\end{tabular}

Table 2. Response according to pretreatment sPD-L1 levels. Response was evaluated in 113 patients. The results are presented as total numbers (percentages). $s P D-L 1$ soluble programmed death ligand-1, ICI immune checkpoint inhibitor, $C R$ complete response, $P R$ partial response, $S D$ stable disease, $P D$ progressive disease. ${ }^{*}$ Significance at $\mathrm{p}<0.05\left(\chi^{2}\right.$ test $)$. 

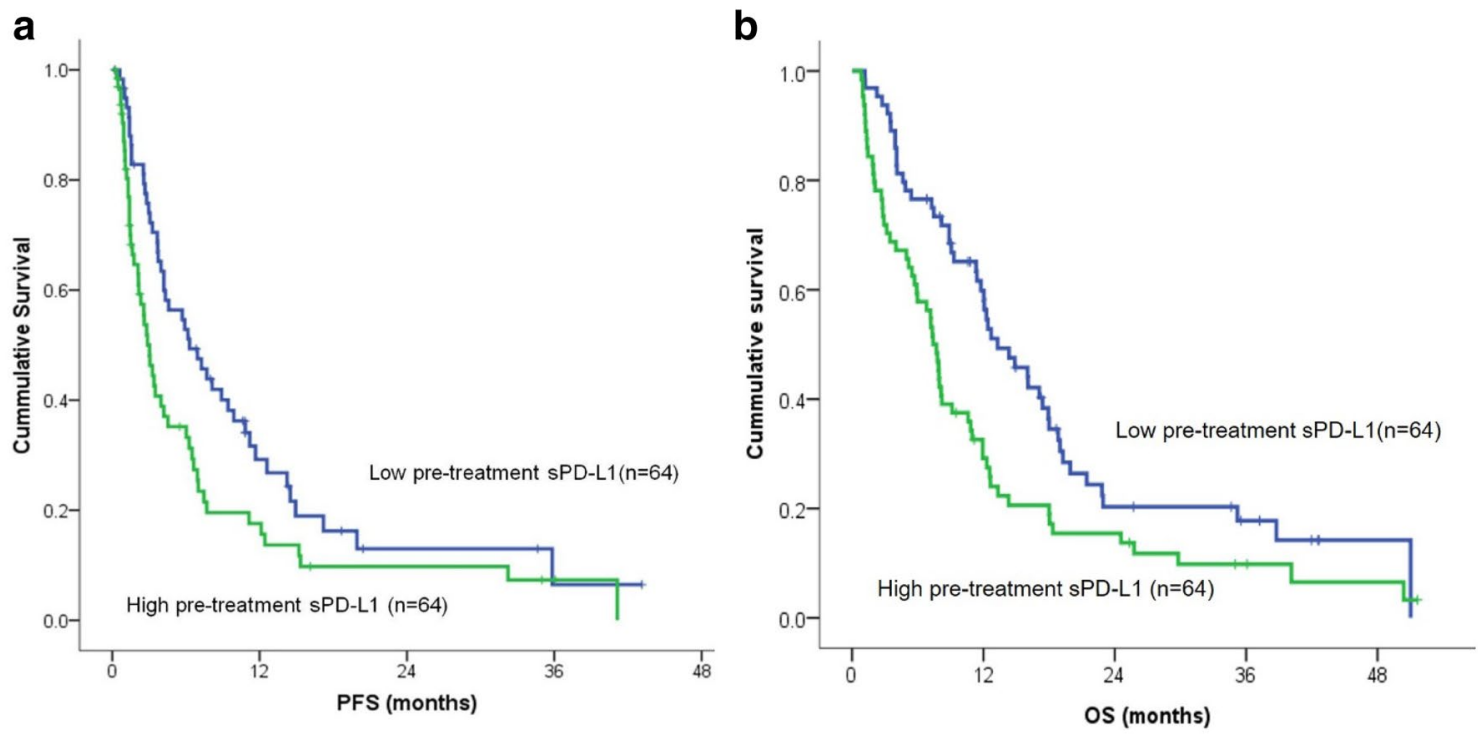

Figure 1. Kaplan-Meier curves for progression-free survival (a) and overall survival (b) stratified by soluble programmed death ligand 1 level. PFS progression-free survival, OS overall survival.

change was quite different among the 'immunogenic' tumor types, such as melanoma, NSCLC, and genitourinary tumors. Possible explanations for these different patterns include different sources of sPD-L1 or carcinomaspecific biological differences. These patterns might also have been affected by differences in sampling intervals; the median sampling interval for NSCLC patients was significantly shorter than the intervals for melanoma or SCLC patients (Supplementary Table 1).

Relationship between changes in SPD-L1 levels and response or prognosis. The analyses to examine relationships between $\triangle$ sPD-L1 and treatment response did not reveal any apparent patterns (Supplementary Fig. 4 and Supplementary Table 2). There was no significant difference in pan-cancer PFS according to $\triangle$ sPD-L1. However, NSCLC patients with a $>100 \%$ increase in sPD-L1 levels after ICI treatment had longer PFS than patients without this increase (Fig. 3a); the median PFS was 6.3 months (95\% CI 0.0-19.4 months) versus 1.2 months (95\% CI 0.6-1.8 months), respectively ( $\mathrm{p}=0.029, \log$-rank test). The opposite was observed in patients with melanoma. The median PFS was 0.9 months (95\% CI not available) versus 5.7 months (95\% CI 3.8-7.6 months) in patients with $\Delta$ SPD-L1 of $100 \%$ or more versus those with $\Delta$ sPD-L1 less than $100 \%$ after ICI treatment $(\mathrm{p}<0.001, \log$-rank test; Fig. $3 \mathrm{~b})$. The OS response was in the same direction as PFS but was not statistically significant in patients with melanoma. In patients with NSCLC, the median OS after ICI treatment was 14.3 months (95\% CI $0.0-30.8$ months) in patients with $\Delta$ SPD-L1 $\geq 100 \%$ versus 5.7 months (95\% CI $0.0-$ 11.4 months) in those with $\Delta$ sPD-L1 $<100 \%$ ( $\mathrm{p}=0.022$, log-rank test; Fig. $3 \mathrm{c})$. The median OS was 3.9 months (95\% CI 2.1-5.7 months) versus 11.4 months (95\% CI 10.6-12.2 months) ( $\mathrm{p}=0.827$, log-rank test) in patients with melanoma (Fig. 3d).

Correlation between SPD-L1 levels and tissue PD-L1 expression or circulating immune cells. We next examined the correlation between tissue PD-L1 expression and SPD-L1 levels. A Spearman's rho value of $0.069(\mathrm{p}=0.575)$ suggested that these two factors were not correlated. The mean sPD-L1 levels in the negative/low PD-L1 expression group were also not significantly different from those in the moderate/high expression group $(12.0 \pm 1.0 \mathrm{pg} / \mu \mathrm{L}$ versus $12.9 \pm 1.2 \mathrm{pg} / \mu \mathrm{L}$, respectively; $\mathrm{p}=0.649, t$ test $)$. We also examined the correlation between SPD-L1 levels and NLR or circulating immune cell numbers (Table 4 and Fig. 4). NLR, white blood cell count, and absolute neutrophil count (ANC) were positively correlated with sPD-L1 levels (Fig. 4a-c). The proportion and total number of lymphocytes were negatively correlated with sPD-L1 levels (Fig. 4e,f).

\section{Discussion}

The results of this study suggest that pretreatment serum sPD-L1 concentrations can be used to predict treatment response, PFS, and OS in patients receiving ICI treatment for advanced solid tumors. We found that a high baseline sPD-L1 level predicted a low disease control rate. The pretreatment SPD-L1 level was an independent prognostic factor predicting PFS and OS, even after controlling for known prognostic variables. Many studies of sPD-L1 have been published in recent years. Most studies have found that high pretreatment sPD-L1 levels are associated with decreased survival in patients with advanced solid tumors (e.g., lung cancer, gastric cancer, renal cell carcinoma, melanoma, hepatocellular carcinoma, pancreatic cancer, and soft tissue sarcoma) ${ }^{31,33,35-43}$. High pretreatment sPD-L1 levels are also associated with a poor response to ICI treatment in patients with melanoma or lung cancer ${ }^{36,44}$. Therefore, it has been generally accepted that high pretreatment sPD-L1 levels are associated with a poor treatment response and a worse prognosis. The results of this study are consistent with 


\begin{tabular}{|c|c|c|c|c|c|c|c|c|c|}
\hline & \multirow[b]{3}{*}{$\mathrm{N}=128$} & \multicolumn{4}{|l|}{ PFS } & \multicolumn{4}{|l|}{ os } \\
\hline & & \multicolumn{2}{|l|}{ Univariate analysis } & \multicolumn{2}{|l|}{ Multivariate analysis } & \multicolumn{2}{|l|}{ Univariate analysis } & \multicolumn{2}{|l|}{ Multivariate analysis } \\
\hline & & $\begin{array}{l}\text { Median (95\% CI) } \\
\text { (months) }\end{array}$ & p value & $\operatorname{Exp}(B)(95 \%$ CI $)$ & p value & $\begin{array}{l}\text { Median }(95 \% \mathrm{CI}) \\
\text { (months) }\end{array}$ & p value & $\operatorname{Exp}(B)(95 \%$ CI $)$ & p value \\
\hline \multicolumn{10}{|l|}{ Age (years) } \\
\hline$<65$ & 78 & $3.4(2.4-4.4)$ & \multirow{2}{*}{0.191} & & & $8.2(5.4-11.0)$ & \multirow{2}{*}{0.387} & & \\
\hline$\geq 65$ & 50 & $7.3(5.8-8.7)$ & & & & $14.9(10.5-19.2)$ & & & \\
\hline \multicolumn{10}{|l|}{ Sex } \\
\hline $\mathrm{M}$ & 89 & $3.7(2.4-5.0)$ & \multirow{2}{*}{0.163} & & & $9.1(6.0-12.3)$ & \multirow{2}{*}{0.122} & & \\
\hline $\mathrm{F}$ & 39 & $6.3(2.4-10.2)$ & & & & $12.1(9.1-15.1)$ & & & \\
\hline \multicolumn{10}{|l|}{ ECOG PS } \\
\hline 0 & 28 & $7.7(0.8-14.6)$ & \multirow{2}{*}{0.012} & \multirow{2}{*}{$1.223(0.560-2.671)$} & \multirow{2}{*}{0.614} & $12.7(0-27.3)$ & \multirow{2}{*}{0.036} & \multirow{2}{*}{$1.402(0.852-2.308)$} & \multirow{2}{*}{0.184} \\
\hline 1 & 100 & $4.0(3.1-4.8)$ & & & & $10.6(7.2-14.0)$ & & & \\
\hline \multicolumn{10}{|l|}{ Radiation therapy } \\
\hline Prior to ICI & 57 & $3.7(2.2-5.2)$ & \multirow[b]{2}{*}{0.056} & & & $8.1(3.4-12.9)$ & \multirow[b]{2}{*}{0.058} & & \\
\hline $\begin{array}{l}\text { After ICI or never irradi- } \\
\text { ated }\end{array}$ & 71 & $6.2(3.3-9.0)$ & & & & $12.3(8.1-15.9)$ & & & \\
\hline \multicolumn{10}{|l|}{ Tissue PD-L1 IHC } \\
\hline Negative, weak & 43 & $3.0(2.4-3.7)$ & \multirow{3}{*}{0.021} & & & $8.2(3.4-13.0)$ & & & \\
\hline Moderate/strong & 25 & $6.9(5.8-8.1)$ & & $2.232(1.119-4.453)$ & 0.023 & $12.6(6.0-19.1)$ & 0.841 & & \\
\hline Unknown & 60 & - & & & & - & & & \\
\hline NLR & & & & & & & & & \\
\hline$<2.8$ & 62 & $5.7(3.8-7.6)$ & 0.042 & $1055(0.531-2099)$ & 0878 & $14.3(9.5-19.2)$ & $<0001$ & $1913(1242-2946)$ & 0003 \\
\hline$\geq 2.8$ & 66 & $3.3(1.8-4.7)$ & 0.042 & $1.055(0.531-2.099)$ & 0.878 & $7.2(4.2-10.1)$ & $<0.001$ & $1.913(1.242-2.946)$ & 0.003 \\
\hline Platelet count & & & & & & & & & \\
\hline$<250 \mathrm{k}$ & 67 & $6.3(3.4-9.2)$ & 0065 & & & $12.4(10.4-14.4)$ & 0016 & $1474(090-2195)$ & 0056 \\
\hline$\geq 250 \mathrm{k}$ & 61 & $3.3(2.0-4.6)$ & 0.065 & & & $7.7(6.7-8.7)$ & 0.016 & $1.4 / 4(0.990-2.195)$ & 0.056 \\
\hline Serum albumin $(\mathrm{g} / \mathrm{dL})$ & & & & & & & & & \\
\hline$<4.0$ & 70 & $2.8(2.1-3.5)$ & 0047 & $1788(0908-3521)$ & 0093 & $6.8(4.3-9.3)$ & 0006 & $1083(0600-1700)$ & 0720 \\
\hline$\geq 4.0$ & 58 & $6.9(5.5-8.4)$ & $0.04 \prime$ & $1.700(0.900-5.321)$ & 0.093 & $14.3(9.9-18.8)$ & 0.000 & $1.083(0.090-1.700)$ & 0.129 \\
\hline Serum total protein $(\mathrm{g} / \mathrm{d}$ & & & & & & & & & \\
\hline$<7.2$ & 65 & $3.4(2.5-4.3)$ & 0078 & & & $8.0(6.5-9.4)$ & 0009 & $1766(1148-2719)$ & 0010 \\
\hline$\geq 7.2$ & 63 & $6.2(2.7-9.7)$ & $0.0 \%$ & & & $12.7(9.6-15.8)$ & 0.009 & $1 . / 00(1.148-2.119)$ & 0.010 \\
\hline Glucose (mg/dL) & & & & & & & & & \\
\hline$<126$ & 85 & $3.7(2.5-4.8)$ & & & & $11.0(7.2-14.8)$ & & & \\
\hline$\geq 126$ & 41 & $6.5(3.3-9.7)$ & 0.124 & & & $12.3(8.1-16.5)$ & 0.808 & & \\
\hline Unknown & 2 & - & & & & - & & & \\
\hline sPD-L1 level (pg/ $\mu \mathrm{L})$ & & & & & & & & & \\
\hline$<11$ & 64 & $6.3(3.0-9.6)$ & 0023 & $1928(1038-3581)$ & 0038 & $13.3(9.2-17.4)$ & 0005 & $1788(1207-2650)$ & 0004 \\
\hline$\geq 11$ & 64 & $2.9(2.1-3.7)$ & 0.023 & $1.920(1.030-5.501)$ & 0.030 & $7.4(6.3-8.5)$ & 0.005 & $1 . / 00(1.207-2.030)$ & 0.004 \\
\hline
\end{tabular}

Table 3. Univariate and multivariate analysis of PFS and OS* PFS progression-free survival, OS overall survival, CI confidence interval, ECOG PS eastern cooperative oncology group performance status, ICI immune checkpoint inhibitor, $P D-L 1$ programmed death ligand-1, IHC immunohistochemical stain, NLR neutrophil to lymphocyte ratio, $s P D-L 1$ soluble programmed death ligand-1. ${ }^{*}$ Significant when $\mathrm{p}$ value is less than 0.05 . Variables with $p<0.05$ were examined in the multivariate analyses.

those of previous studies in that pretreatment sPD-L1 levels had predictive and prognostic value for patients with advanced cancer.

We also investigated whether changes in SPD-L1 levels after ICI treatment are associated with response or prognosis. We were not able to acquire the sample sizes required to identify correlations between the extent of SPD-L1 changes and tumor response or PFS. Because each tumor type had very different patterns of change in sPD-L1 levels, the effects of changes in SPD-L1 on PFS were likely diluted. This possibility was supported by our observation of the opposite pattern when each cancer type (melanoma and NSCLC) was analyzed separately. Few studies have examined the relationships between SPD-L1 dynamics and prognosis in patients receiving radiation or chemoradiation, and the results of those limited studies have been inconsistent. Increased sPD-L1 levels after chemoradiation are associated with a poor prognosis in patients with rectal cancer ${ }^{45}$. In contrast, preliminary results indicate that in patients with biliary tract cancer, increased SPD-L1 levels after chemotherapy are associated with longer $\mathrm{PFS}^{46}$. In patients with advanced pancreatic cancer receiving cytotoxic chemotherapy, sPD-L1 dynamics correlate with the disease cours $\mathrm{e}^{39}$. Several studies have been performed to investigate the dynamics of sPD-L1 changes over time in patients receiving ICI treatment. One study of patients receiving ipilimumab-based 

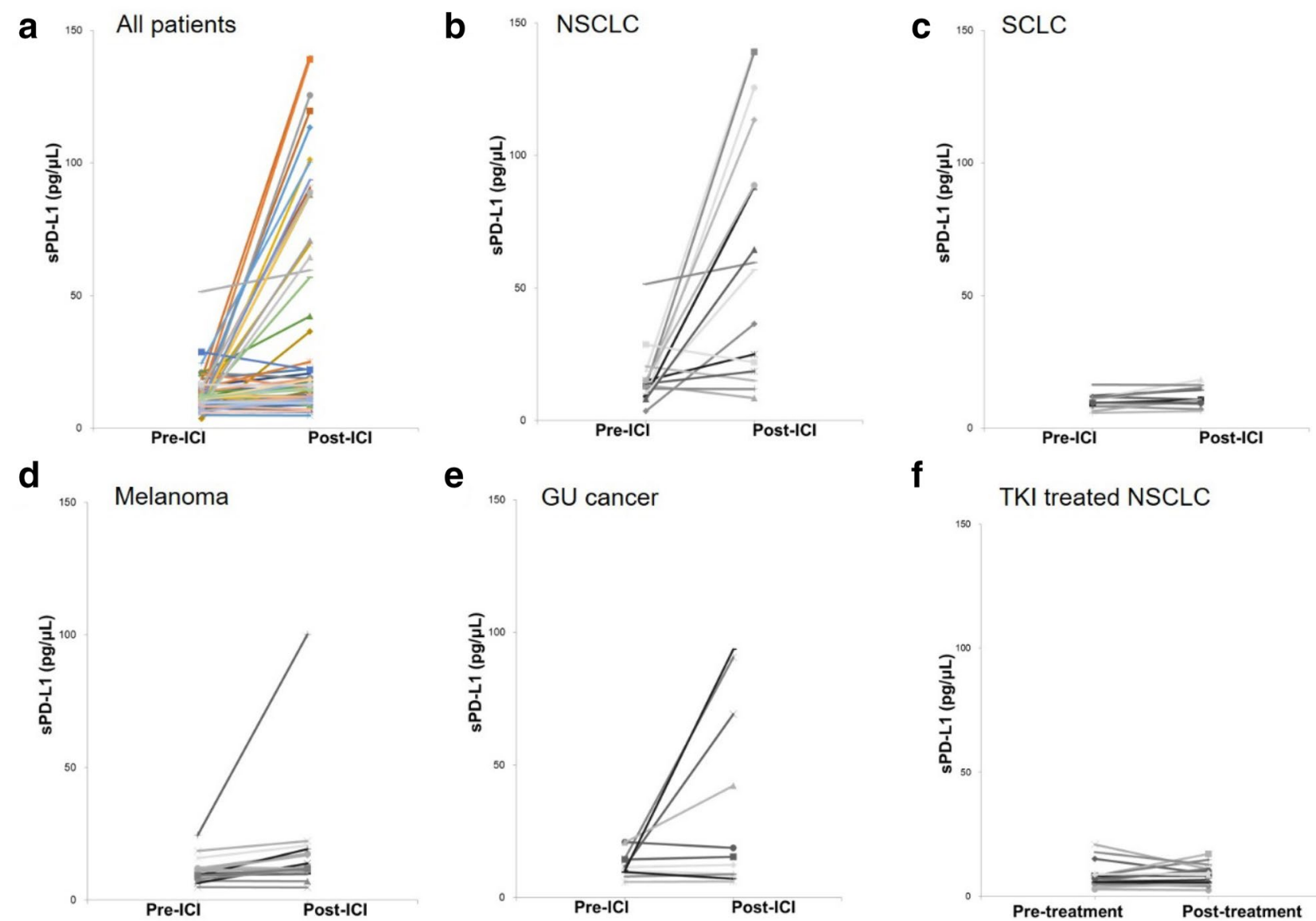

Figure 2. Changes in sPD-L1 levels before and after treatment, according to cancer type and treatment type in all patients (a), NSCLC patients (b), SCLC patients (c), melanoma patients (d), GU cancer patients (e), and NSCLC patients treated with TKIs (f). The median time points for post-ICI sampling were 15 days (NSCLC), 21 days (melanoma and GU cancer), and 42 days (SCLC). Pre-ICI before administration of immune checkpoint inhibitor, Post-ICI after administration of immune checkpoint inhibitor, NSCLC non-small cell lung cancer, $S C L C$ small cell lung cancer, GU genitourinary, TKI tyrosine kinase inhibitor.

treatment for advanced melanoma found that many who had a $\geq 1.5$-fold increase in sPD-L1 within 4.5 months after treatment experienced progressive disease ${ }^{44}$. A study analyzed PD-L1 mRNA expression in plasma-derived exosomes in melanoma and NSCLC patients at baseline and 2 months after PD-1 inhibitor treatment. They showed that exosomal mRNA copies of PD-L1 were correlated with tumor response in melanoma $(\mathrm{n}=18)$ and $\operatorname{NSCLC~}(n=8)$ patients ${ }^{47}$. Another study of 21 patients with lung, gastric, or bladder cancer who underwent anti-PD-1 therapy found that a reduction in plasma SPD-L1 levels is significantly correlated with tumor size reduction ${ }^{48}$. The significance of SPD-L1 in our NSCLC population was inconsistent with other studies. It seems too early to draw conclusions, as the sample size of all previously published studies was very small.

We also examined the association between PD-L1 expression levels in tumor tissue and circulating sPD-L1 levels. PD-L1 expression in tumor tissue did not correlate with SPD-L1 levels. This was an unexpected finding, as we expected the levels of sPD-L1 to reflect the expression of tissue PD-L1. Therefore, we explored circulating immune cells as a possible source of SPD-L1. sPD-L1 levels were positively correlated with NLR and negatively correlated with total lymphocyte numbers. The results of previous studies suggest that SPD-L1 originates mainly from a membrane-bound form of PD-L1 present in cancer cells or immune cells ${ }^{37,43,49}$. The results of our study suggest that SPD-L1 is derived from cells identified as neutrophils in routine complete blood count tests. Additionally, in a previous study that analyzed peripheral immune cells from 28 cancer patients, 5 to $35 \%$ of peripheral blood myeloid-derived suppressor cells (MDSCs) expressed PD-L1. The expression of PD-L1 is highest in granulocytic MDSCs $(35.8 \%)$ and lower in T cells $(<1 \%)$, most NK cells $(<1 \%)$, and B cells $(11 \%)^{50}$. Therefore, we can carefully assume that SPD-L1 originates from granulocytic MDSCs. Considering the limited information currently available, however, the main source of sPD-L1 should be explained in future studies.

This study had some limitations. The study population was heterogeneous with respect to cancer type, blood sampling times, ICI administration timing, and post-ICI treatment regimens. This heterogeneity might have reduced the power to detect the effects of various characteristics for individual types of cancer. There were also no reference levels for sPD-L1, and the results between assay kits seemed to be quite different. There are also no pre-established cutoff levels that predict response or prognosis. To overcome this problem, some researchers are investigating reproducible, standardizable methods that can be used instead of ELISA ${ }^{49}$. 


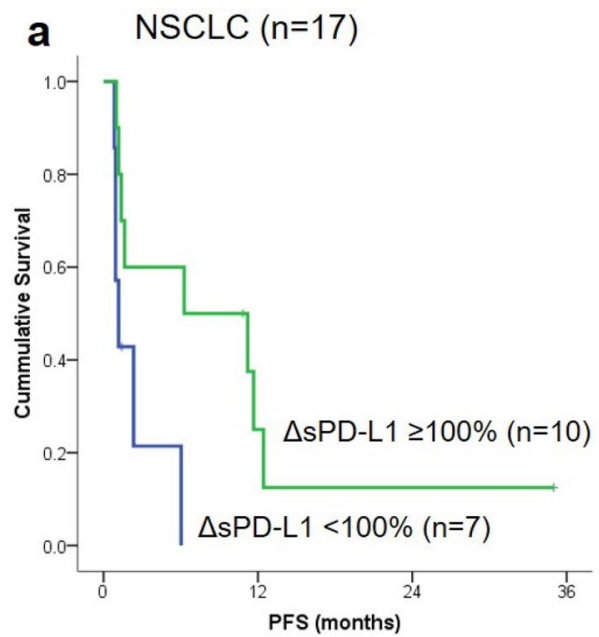

$$
\text { b Melanoma }(n=15)
$$
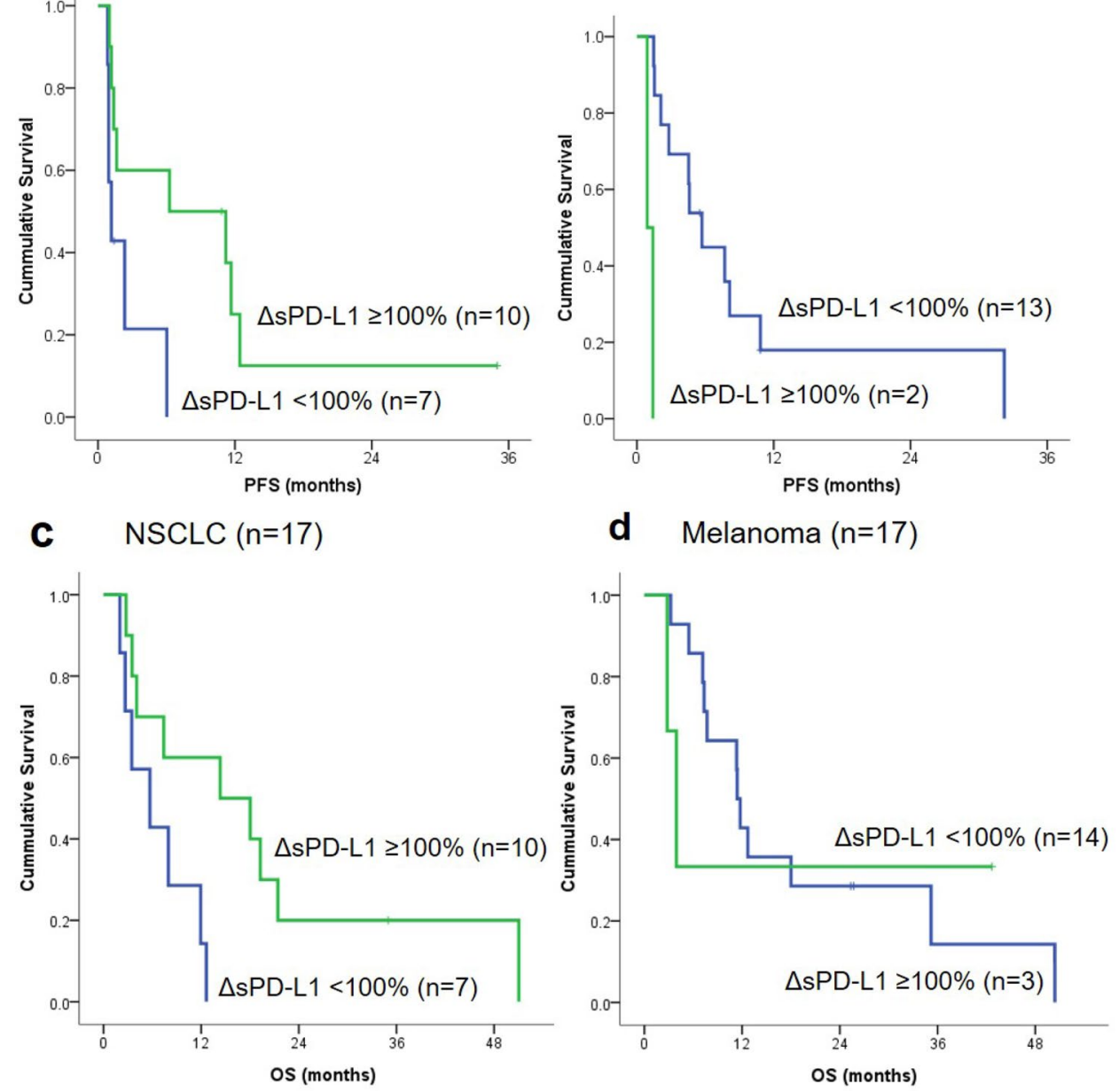

Figure 3. Kaplan-Meier curves for progression-free survival and overall survival in patients with NSCLC (a,c) and patients with melanoma (b,d) stratified by soluble programmed death ligand 1 levels. NSCLC non-small cell lung cancer, $\triangle s P D-L 1$ change in SPD-L1 concentration between pretreatment and posttreatment sampling, PFS progression-free survival, OS overall survival.

\begin{tabular}{|l|c|c|}
\hline & Pearson's correlation coefficient & p value $^{*}$ \\
\hline NLR & 0.309 & $<0.001$ \\
\hline WBC & 0.202 & 0.023 \\
\hline ANC & 0.183 & 0.040 \\
\hline Neutrophil & 0.081 & 0.369 \\
\hline Lymphocyte & -0.277 & 0.002 \\
\hline ALC & -0.222 & 0.012 \\
\hline
\end{tabular}

Table 4. Analysis of correlations between sPD-L1 and circulating immune cells. Two outliers with extremely high sPD-L1 levels or absolute neutrophil counts were excluded from these analyses. $S P D-L 1$ soluble programmed death ligand-1, NLR neutrophil to lymphocyte ratio, $W B C$ white blood cell count, $A N C$ absolute neutrophil count, $A L C$ absolute lymphocyte count. ${ }^{\star}$ Significance at $\mathrm{p}<0.05$.

In summary, high pretreatment sPD-L1 levels were associated with low disease control rates. sPD-L1 levels were an independent predictor of PFS and OS in patients receiving ICI treatment for advanced cancer. sPD-L1 was likely derived from peripheral blood neutrophils, and levels generally increased following ICI administration. The amplitude of sPD-L1 change after ICI treatment was associated with PFS in patients with NSCLC and melanoma but in the opposite direction for each cancer type. A future, larger study should be undertaken to reveal the significance of changes in SPD-L1 levels for each carcinoma type. 

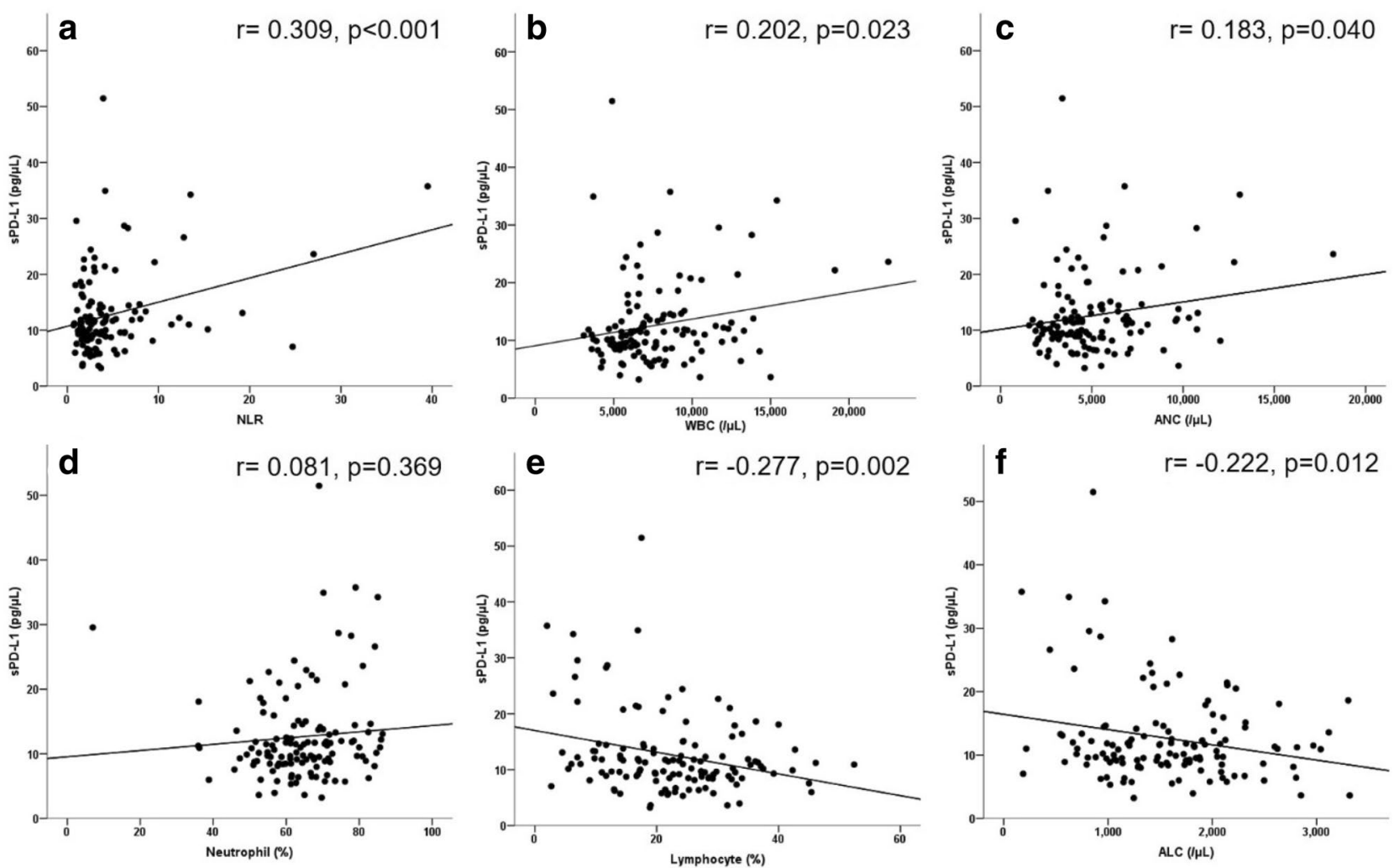

Figure 4. Correlation between sPD-L1 levels and circulating immune cells, including NLR (a), WBCs (b), ANCs (c), neutrophils (d), lymphocytes (e), and ALCs (f). Pearson's correlation coefficients are denoted as $\mathrm{r}$. $N L R$ neutrophil to lymphocyte ratio, $W B C$ white blood cell, $A N C$ absolute neutrophil count, $A L C$ absolute lymphocyte count.

\section{Methods}

Patients. Blood samples were taken from each cancer patient before they received ICI treatment. Some patients participated in other, previously published studies ${ }^{51-53}$. Posttreatment samples were obtained from 67 patients at either the next visit or a later visit. Patients were eligible for the study if they (1) were 18 years of age or older, (2) had a histologically confirmed malignancy, (3) received ICI treatment at Seoul National University Hospital, (4) had study samples taken before and/or after ICI treatment, and (5) completed a written consent form for research using human derivatives, which allowed for secondary utilization of samples (IRB No. 1104086-359). A patient was excluded from the study if they had a diagnosis of two or more types of malignancy within the previous 5 years, withdrew consent before or during the study, or not enough samples were stored for analysis. Retrospective clinical and follow-up information was obtained from the medical records. Pre- and posttreatment samples from patients who received molecularly targeted agents were also analyzed. Blood sampling and analyses were performed after the study protocol was approved by the Institutional Review Board. All patients provided written informed consent to participate in this study. The study protocol was approved by the Institutional Review Board of Seoul National University Hospital (IRB No. 2002-070-110). All study procedures were performed in accordance with the Helsinki Declaration and its later amendments or comparable ethical standards.

SPD-L1 ELISA. Serum was obtained by centrifugation $(1300 \times \mathrm{g}$ for $10 \mathrm{~min})$ and then aliquoted and stored at $-80{ }^{\circ} \mathrm{C}$ until study analysis. sPD-L1 was assayed using a commercially available ELISA Kit (BMS2212, Invitrogen, Vienna, Austria) following the manufacturer's instructions. Samples were analyzed in duplicate for each marker.

Statistical analysis. Demographic and clinical parameters were analyzed using descriptive statistics. Differences in SPD-L1 distribution and median values in healthy donors and cancer patients were compared using nonparametric Mann-Whitney $U$ tests. ROC curve analysis was used to determine the optimal sPD-L1 cutoff point for predicting treatment resistance. Relationships between treatment response and SPD-L1 or clinical variables were analyzed using $\chi^{2}$-tests and Student's $t$ tests. Kaplan-Meier survival analyses and Cox proportional hazards models were used to analyze PFS and OS. Analyses of correlations between sPD-L1 and circulating immune cells were performed by calculating Pearson correlation coefficients because the data met the assumptions of a normal distribution. Because the data for tissue PD-L1 expression did not follow a normal distribution, correlations with sPD-L1 were assessed using the nonparametric Spearman's rho method. Statistical analyses 
were performed and graphics generated using IBM SPSS statistics v.21 (IBM, Armonk, NY, USA) and Excel 2019 (Microsoft, Redmond, WA, USA) software. A p value $<0.05$ was considered statistically significant.

\section{Data availability}

The datasets generated and/or analyzed in the current study are available from the corresponding author upon reasonable request.

Received: 26 October 2020; Accepted: 1 June 2021

Published online: 05 October 2021

\section{References}

1. Marin-Acevedo, J. A., Chirila, R. M. \& Dronca, R. S. in Mayo Clinic Proceedings 1321-1329 (Elsevier).

2. Duffy, M. J. \& Crown, J. Biomarkers for predicting response to immunotherapy with immune checkpoint inhibitors in cancer patients. Clin. Chem. 65, 1228-1238 (2019).

3. Borghaei, H. et al. Nivolumab versus docetaxel in advanced nonsquamous non-small-cell lung cancer. N. Engl. J. Med. 373, 1627-1639 (2015).

4. Carbognin, L. et al. Differential activity of nivolumab, pembrolizumab and MPDL3280A according to the tumor expression of programmed death-ligand-1 (PD-L1): Sensitivity analysis of trials in melanoma, lung and genitourinary cancers. PLoS One 10, e0130142 (2015).

5. Herbst, R. S. et al. Pembrolizumab versus docetaxel for previously treated, PD-L1-positive, advanced non-small-cell lung cancer (KEYNOTE-010): A randomised controlled trial. Lancet 387, 1540-1550 (2016).

6. Mu, C.-Y., Huang, J.-A., Chen, Y., Chen, C. \& Zhang, X.-G. High expression of PD-L1 in lung cancer may contribute to poor prognosis and tumor cells immune escape through suppressing tumor infiltrating dendritic cells maturation. Med. Oncol. 28, 682-688 (2011).

7. Pawelczyk, K. et al. Role of PD-L1 expression in non-small cell lung cancer and their prognostic significance according to clinicopathological factors and diagnostic markers. Int. J. Mol. Sci. 20, 824 (2019).

8. Wang, A. et al. The prognostic value of PD-L1 expression for non-small cell lung cancer patients: a meta-analysis. Eur. J. Surg. Oncol. (EJSO) 41, 450-456 (2015).

9. Hirsch, F. R. et al. PD-L1 immunohistochemistry assays for lung cancer: Results from phase 1 of the blueprint PD-L1 IHC assay comparison project. J. Thorac. Oncol. 12, 208-222 (2017).

10. McLaughlin, J. et al. Quantitative assessment of the heterogeneity of PD-L1 expression in non-small-cell lung cancer. JAMA Oncol. 2(1), 46-54 (2016).

11. Rimm, D. L. et al. A prospective, multi-institutional, pathologist-based assessment of 4 immunohistochemistry assays for PD-L1 expression in non-small cell lung cancer. JAMA Oncol. 3, 1051-1058 (2017).

12. Hewish, M., Lord, C. J., Martin, S. A., Cunningham, D. \& Ashworth, A. Mismatch repair deficient colorectal cancer in the era of personalized treatment. Nat. Rev. Clin. Oncol. 7, 197 (2010).

13. Sargent, D. J. et al. (American Society of Clinical Oncology, 2014).

14. Marcus, L., Lemery, S. J., Keegan, P. \& Pazdur, R. FDA approval summary: Pembrolizumab for the treatment of microsatellite instability-high solid tumors. Clin. Cancer Res. 25, 3753-3758 (2019).

15. Park, K. et al. Pan-Asian adapted ESMO Clinical Practice Guidelines for the management of patients with locally-advanced unresectable non-small-cell lung cancer: a KSMO-ESMO initiative endorsed by CSCO, ISMPO, JSMO, MOS, SSO and TOS. Ann. Oncol. 31, 191-201. https://doi.org/10.1016/j.annonc.2019.10.026 (2020).

16. Planchard, D. et al. Metastatic non-small cell lung cancer: ESMO Clinical Practice Guidelines for diagnosis, treatment and followup. Ann. Oncol. 30, 863-870 (2019).

17. Bigras, G. et al. Small biopsies misclassify up to $35 \%$ of PD-L1 assessments in advanced lung non-small cell lung carcinomas. Appl. Immunohistochem. Mol. Morphol. 26, 701-708 (2018).

18. Anantharaman, A. et al. Programmed death-ligand 1 (PD-L1) characterization of circulating tumor cells (CTCs) in muscle invasive and metastatic bladder cancer patients. BMC Cancer 16, 744 (2016).

19. Chae, Y. K. et al. Clinical implications of circulating tumor DNA tumor mutational burden (ctDNA TMB) in non-small cell lung cancer. Oncologist 24, 820-828 (2019).

20. Nicolazzo, C. et al. Monitoring PD-L1 positive circulating tumor cells in non-small cell lung cancer patients treated with the PD-1 inhibitor Nivolumab. Sci. Rep. 6, 31726 (2016).

21. Sanmamed, M. et al. Changes in serum interleukin-8 (IL-8) levels reflect and predict response to anti-PD-1 treatment in melanoma and non-small-cell lung cancer patients. Ann. Oncol. 28, 1988-1995 (2017).

22. Weber, J. S. et al. A serum protein signature associated with outcome after anti-PD-1 therapy in metastatic melanoma. Cancer Immunol. Res. 6, 79-86 (2018).

23. Nixon, A. B. et al. Peripheral immune-based biomarkers in cancer immunotherapy: Can we realize their predictive potential?. J. Immunother. Cancer 7, 1-14 (2019).

24. Kwon, M. et al. PD-1 blockade reinvigorates bone marrow $\mathrm{CD}^{+} \mathrm{T}$ cells from patients with multiple myeloma in the presence of TGF $\beta$ inhibitors. Clin. Cancer Res. 26, 1644-1655 (2020).

25. Im, S. J. et al. Defining CD8 ${ }^{+}$T cells that provide the proliferative burst after PD-1 therapy. Nature 537, 417-421 (2016).

26. Mazzaschi, G. et al. The circulating pool of functionally competent NK and CD8 ${ }^{+}$cells predicts the outcome of anti-PD1 treatment in advanced NSCLC. Lung Cancer (Amsterdam, Netherlands) 127, 153-163 (2019).

27. Hassounah, N. B. et al. Identification and characterization of an alternative cancer-derived PD-L1 splice variant. Cancer Immunol. Immunother. 68, 407-420. https://doi.org/10.1007/s00262-018-2284-z (2019).

28. Gong, B. et al. Secreted PD-L1 variants mediate resistance to PD-L1 blockade therapy in non-small cell lung cancer. J. Exp. Med. 216, 982-1000 (2019).

29. Mahoney, K. M. et al. A secreted PD-L1 splice variant that covalently dimerizes and mediates immunosuppression. Cancer Immunol. Immunother. 68, 421-432 (2019).

30. Rossille, D. et al. High level of soluble programmed cell death ligand 1 in blood impacts overall survival in aggressive diffuse large B-Cell lymphoma: results from a French multicenter clinical trial. Leukemia 28, 2367 (2014).

31. Finkelmeier, F. et al. High levels of the soluble programmed death-ligand (sPD-L1) identify hepatocellular carcinoma patients with a poor prognosis. Eur. J. Cancer 59, 152-159 (2016).

32. Ito, M. et al. Is high serum programmed death ligand 1 level a risk factor for poor survival in patients with gastric cancer?. Ann. Gastroenterol. Surg. 2, 313-318 (2018).

33. Zhang, J. et al. Circulating PD-L 1 in NSCLC patients and the correlation between the level of PD-L 1 expression and the clinical characteristics. Thorac. Cancer 6, 534-538 (2015). 
34. Mazzaschi, G. et al. Soluble PD-L1 and circulating CD8 $8^{+} \mathrm{PD}-1^{+}$and NK cells enclose a prognostic and predictive immune effector score in immunotherapy treated NSCLC patients. Lung Cancer (Amsterdam, Netherlands) 148, 1-11 (2020).

35. Asanuma, K. et al. Soluble programmed death-ligand 1 rather than PD-L1 on tumor cells effectively predicts metastasis and prognosis in soft tissue sarcomas. Sci. Rep. 10, 1-9(2020).

36. Dronca, R. S. et al. Association of soluble PD-L1 (sPD-L1) with decreased survival in metastatic melanoma. J. Clin. Oncol. 35(7) suppl, 4 (2017).

37. Frigola, X. et al. Identification of a soluble form of $\mathrm{B} 7-\mathrm{H} 1$ that retains immunosuppressive activity and is associated with aggressive renal cell carcinoma. Clin. Cancer. Res. 17, 1915-1923 (2011).

38. Okuma, Y. et al. High plasma levels of soluble programmed cell death ligand 1 are prognostic for reduced survival in advanced lung cancer. Lung Cancer 104, 1-6 (2017).

39. Park, H. et al. Prognostic implications of soluble programmed death-ligand 1 and its dynamics during chemotherapy in unresectable pancreatic cancer. Sci. Rep. 9, 11131. https://doi.org/10.1038/s41598-019-47330-1 (2019).

40. Shigemori, T. et al. Soluble PD-L1 expression in circulation as a predictive marker for recurrence and prognosis in gastric cancer: direct comparison of the clinical burden between tissue and serum PD-L1 expression. Ann. Surg. Oncol. 26, 876-883 (2019).

41. Sideras, K. et al. Circulating levels of PD-L1 and Galectin-9 are associated with patient survival in surgically treated Hepatocellular Carcinoma independent of their intra-tumoral expression levels. Sci. Rep. 9, 10677. https://doi.org/10.1038/s41598-019-47235-z (2019).

42. Zhao, J. et al. Plasma levels of soluble programmed death ligand-1 may be associated with overall survival in nonsmall cell lung cancer patients receiving thoracic radiotherapy. Medicine (Baltimore) 96, e6102-e6102. https://doi.org/10.1097/MD.0000000000 006102 (2017).

43. Zhu, X. \& Lang, J. Soluble PD-1 and PD-L1: Predictive and prognostic significance in cancer. Oncotarget 8, 97671-97682. https:// doi.org/10.18632/oncotarget.18311 (2017).

44. Zhou, J. et al. Soluble PD-L1 as a biomarker in malignant melanoma treated with checkpoint blockade. Cancer Immunol. Res. 5, 480-492 (2017).

45. Tominaga, T. et al. Clinical significance of soluble programmed cell death-1 and soluble programmed cell death-ligand 1 in patients with locally advanced rectal cancer treated with neoadjuvant chemoradiotherapy. PLoS One 14, e0212978 (2019).

46. Kim, J. W. et al. The prognostic role of soluble transforming growth factor- $\beta$ related with soluble programmed death-ligand 1 in biliary tract cancer. J. Clin. Oncol. 37(15), suppl. 4094 (2019).

47. Del Re, M. et al. PD-L1 mRNA expression in plasma-derived exosomes is associated with response to anti-PD-1 antibodies in melanoma and NSCLC. Br. J. Cancer 118, 820-824 (2018).

48. Ando, K. et al. Plasma levels of soluble PD-L1 correlate with tumor regression in patients with lung and gastric cancer treated with immune checkpoint inhibitors. Anticancer Res. 39, 5195-5201. https://doi.org/10.21873/anticanres.13716 (2019).

49. Chen, Y. et al. Development of a sandwich ELISA for evaluating soluble PD-L1 (CD274) in human sera of different ages as well as supernatants of PD-L1+ cell lines. Cytokine 56, 231-238 (2011).

50. Donahue, R. N. et al. Analyses of the peripheral immunome following multiple administrations of avelumab, a human IgG1 antiPD-L1 monoclonal antibody. J. Immunother. Cancer 5, 1-16 (2017).

51. Heo, J. Y. et al. The efficacy of immune checkpoint inhibitors in anaplastic lymphoma kinase-positive non-small cell lung cancer. Thorac. Cancer 10, 2117-2123 (2019).

52. Kim, Y.-J. et al. A phase II study of pembrolizumab and paclitaxel in patients with relapsed or refractory small-cell lung cancer. Lung Cancer (Amsterdam, Netherlands) 136, 122-128 (2019).

53. Infante, J. R. et al. A phase Ib dose escalation study of the OX40 agonist MOXR0916 and the PD-L1 inhibitor atezolizumab in patients with advanced solid tumors. J. Clin. Oncol. 34, 101-101, suppl. 101 (2016).

\section{Acknowledgements}

The authors thank the staff of the Cancer Research Institute of Seoul National University College of Medicine for assistance with this study. Su Jeong Heo helped collect and store the clinical samples, Jahyang Choi provided ELISA technical support, and Juyeon Kim assisted with clinical data collection.

\section{Author contributions}

S.Y.O. and T.M.K. designed the study. S.K. performed and analyzed the experiments. S.Y.O. performed the analyses and wrote the first version of the manuscript. T.M.K., B.K., and S.K. provided considerable advice on data interpretation. D.S.H. and D.W.K. substantively revised the manuscript. All authors reviewed the manuscript and approved the final version.

\section{Funding}

This study was supported by grants from the Korean Health Technology R\&D Project "Strategic Center of Cell and Bio Therapy for Heart, Diabetes and Cancer" through the Korea Health Industry Development Institute (KHIDI), funded by the Ministry of Health and Welfare (MHW), Republic of Korea (grant number: HI-17C-2085).

\section{Competing interests}

The authors declare no competing interests.

\section{Additional information}

Supplementary Information The online version contains supplementary material available at https:/doi.org/ 10.1038/s41598-021-99311-y.

Correspondence and requests for materials should be addressed to D.S.H.

Reprints and permissions information is available at www.nature.com/reprints.

Publisher's note Springer Nature remains neutral with regard to jurisdictional claims in published maps and institutional affiliations. 
(c) (i) Open Access This article is licensed under a Creative Commons Attribution 4.0 International cc) License, which permits use, sharing, adaptation, distribution and reproduction in any medium or format, as long as you give appropriate credit to the original author(s) and the source, provide a link to the Creative Commons licence, and indicate if changes were made. The images or other third party material in this article are included in the article's Creative Commons licence, unless indicated otherwise in a credit line to the material. If material is not included in the article's Creative Commons licence and your intended use is not permitted by statutory regulation or exceeds the permitted use, you will need to obtain permission directly from the copyright holder. To view a copy of this licence, visit http://creativecommons.org/licenses/by/4.0/.

(C) The Author(s) 2021 